\title{
Real-time statistical image and video processing for remote sensing and surveillance applications
}

\author{
Mohammad R. Khosravi ${ }^{1} \cdot$ Pooya Tavallali $^{2}$ \\ Published online: 3 September 2021 \\ (c) The Author(s), under exclusive licence to Springer-Verlag GmbH Germany, part of Springer Nature 2021
}

\section{Introduction}

We are pleased to provide the topical collection on "RealTime Statistical Image and Video Processing for Remote Sensing and Surveillance Applications" for Journal of RealTime Image Processing. The real-time processing aims at achieving a computation method for a specific task that can be processed fast enough for human acceptance and interaction. For example, a very well-known and popular machine learning method is the real-time face detection presented in [1]. The method could achieve a face detection ratio of 15-20 frames per second and was used in mobile devices for several years. Similar approaches were later used for various tasks of object detection [2,3]. The real-timeliness of an algorithm or method is especially very useful for various reasons such as real-time decision making or time-critical tasks such as surveillance applications. In such tasks, a user is interested in checking the outputs of a surveillance device or applications. Most real-time approaches presented since 2000 were based on conventional machine learning models such as Ensembles, Boosting and Forest models. Recently, the Deep Learning models have achieved great success in terms of accuracy and performance. Specifically, such models are successfully used in image and video processing tasks. However, these models are very dense and energy consuming due to high complexity of their parameters. Additionally, they occasionally lack the speed needed for their desired tasks. As a result, in recent years, there has been much research focus on reducing the complexity of Deep

Mohammad R. Khosravi

mohammadr.khosravi@iran.ir

Pooya Tavallali

ptavallali@ucmerced.edu

1 Department of Computer Engineering, Persian Gulf University, Bushehr, Iran

2 Department of Electrical Engineering and Computer Science (EECS), University of California, Merced, USA
Learning models and essentially improving their speed while preserving their accuracy. As a result, a very fundamental and hot topic is the application of such models in image and video processing tasks such as remote sensing. This special issue specifically focuses on real-time statistical methods for the above-mentioned tasks. Statistical methods essentially consist of any approach that can learn the relation between a given input and output. Such learned relationship can potentially be used at the test phase for prediction, detection and interpolation purposes. Although deep learning models are a specific part of statistical models, the literature recently separates them from conventional and non-deep learning algorithms. Here, the focus is placed on remote sensing and surveillance applications. The accepted papers of this special issue consist of two types of general topics.

The first group subsumes papers covering statistical computation methods and the second group consists of papers covering deep learning and neural network techniques. The organization of this issue is done based on first reviewing the statistical approaches and then reviewing the deep learning models.

\section{Conventional methods of statistical computing}

The conventional methods of statistical computing consists of methods that do not use deep learning models and usually involve well-known optimization techniques. Such models consist of Hidden Markov Models, Decision Trees, Forest, Boosting, Support Vector Machines, etc. These models are very well developed in the literature of statistical learning and have well understood and interpretable optimization techniques. The conventional methods in the current form have existed for nearly 40 years and achieved a great attention from researchers in the field of Machine Learning since 2000. The following section is reviewing all accepted papers retained for this special issue. 
The authors in [4] present a new framework to satisfy real-time processing requirements in remote sensing (RS) applications. While most existing algorithms have focused on reducing the computational complexity of existing methods, the authors in [4] propose a novel Majorization-Minimization approach, which optimizes a simpler surrogate objective function to approximate the solution for the difficult original problem. The efficacy of the proposed algorithm was presented in real-time applications and are compared with other existing state-of-the-art methods. The proposed surrogate objective function is a converted version of the corresponding non-differentiable objective function to a smoothed one. Finally, the method presented in [4] enhances the applicability of sparsity-driven SAR imaging for realtime RS applications.

The research in [5] tackled the task of automated video highlight generation in large datasets. The authors presented a new unsupervised video highlight generation based on a skimming-based method. The method essentially consisted of clustering techniques for extracting dynamic features from a stream of image frames (videos). The feature is based on analyzing each frame at the pixel level, while also using dense optical flow of the consecutive frames. The approach mainly adopts dual streams for simultaneously processing features of both streams. The presented approach can be used for efficient storage, quick browsing, and retrieval of large collection of video data without losing important aspects for movies and sports video feeds.

A motion-blurred star image restoration algorithm was proposed in [6]. The authors take high dynamic conditions into account for the simulations. The algorithm consists of calculating the coordinate variation of the star spot between adjacent star images from one imaging plane. A motion recursive model is used. Finally, by taking inertial measurement error and the motion model's error with respect to the coordinate, the coordinate variation is corrected. The method was compared with several other existing methods in the literature.

The authors in [7] propose a real-time approach for secret sharing of sensitive remote sensing data. The problem consists of two phases. First, encryption of a multidimensional remote sensing image is performed and then, secure transmission of the image is done, while at the same time ensuring the security, efficiency, and realtime behavior of the algorithm. Additionally, the authors showed that the algorithm is immune to cheating (any kind of manipulation or hacking). The approach consists of extracting the halftone bit planes from the image. Subsequently, these halftone bit planes are pre-processed for producing several copies to be shared with the receivers. At the reconstruction time, the images are regenerated by stacking the shares. Most of the processing time for the algorithm is spent on codebook generation, which the authors have automated by proposing a novel matrix creation process. Finally, the method is parallelized to achieve further reduction of processing time.

The authors of [8] have provided a systematic review of real-time underwater image enhancement. One of the environments being researched and observed continuously by researchers is underwater locations. However, the quality of images provided by underwater surveillance cameras is low according to specific underwater artefacts. Such artefacts range from color filtering to other effects originating from poor quality of sensors. Scattering and absorption are some of the examples that cause the light to be attenuated in underwater; thus, affecting the quality of images and videos. Therefore, one of the main issues with underwater images and videos is recognizing the background of the objects in the images.

The authors of [9] investigated automatic image registration of different remote sensing images of the same scene. The main problem in this task is that different images of the same scene usually have different spatial resolutions. The authors proposed an accurate and efficient method for registration of two images. The proposed method uses a probabilistic model that calculates the probability of different mappings between two images and selects the mapping with highest probability. The registration consisted of three stages, stage one, the pixels are partitioned into two sets and calculates the affine transformation; stage two: potential pixels for matching are selected and in the third stage, a Markov Chain model is trained based on the approximations of the first two stages.

Paper [10] focuses on real-time segmentation of high-resolution remote sensing images. The authors suggest a novel unsupervised method to segment the image. The approach consists of three stages. The first stage partitions the image into equal sized blocks. Each block is then represented by the mean of their R, G, and B components of pixels within the block. Using a clustering approach, the blocks are segmented. The second stage consists of learning a Bayesian approach to refine the achieved segmentation. In the third stage, a graph-based approach is used to detect the regions with complex textures.

The authors of [11] investigated the problem of dehazing an image by segmentation of super pixels. The task is important, because quality of images are affected by hazing and fogging. The authors applied several stages of statistical and machine learning techniques to dehaze the input image in a sequential method. In [12], an environmental application of remote sensing image processing has been provided to control water resources. The authors have reviewed various forms of computational methods and finally proposed their finding including a detection method with block division strategy based on the map-reduce structure. The proposed technique helps to locate abnormal changes surrounding a 
lake (or another form of water reservoir) made by activities of human being.

\section{Deep computing and neural networks}

Paper [13] tackles the issue of prediction in spatial raster time series. Such data are extracted from satellite imagery. The decision-making task on such data is time-consuming because of redundant and parallel computations; thus, fast and real-time processing of such data for geo-spatial events is very important. The difficulty of such a task is due to scalability of existing methods and the high training time for existing Neural-Net based algorithms. The authors propose a new type of recurrent Neural-Net model called ContRast. The proposed model tackles the mentioned issues by removing the necessity for offline adjustments of the network structure, reducing the optimization complexity using single-pass parameter learning mechanism, and reducing the redundant learning. The model is autonomous in learning for real-time prediction of remote sensing data.

The authors of [14] tackle the issue of efficiency in management and classification of remote sensing images by proposing an algorithm based on distributed storage. The authors use the HBase distributed database to store the data and finally train their U-Net over the stored data. They essentially take advantage of distributed computing with NeuralNets to train their network for the dataset.

In [15], author focuses on the problem of feature detection in circular harmonic spectra in two-dimensional images. Examples of such a problem consists of understanding objects that do not change shape but appear differently when seen from different perspectives. The problem becomes obvious considering that machines understand environment through images that are expressed in a Cartesian space, although objects may be more intuitively described in polar coordinates. Solving such a problem is very complex and time consuming. The author in [15] proposed a deep learning-based approach for solving such problems using polarseparable responses of filter banks.

Paper [16] proposes a real-time neural network model that over-comes the shortcomings of real-time surveillance of remote sensing images, especially with multi-spectral images of high resolution. To achieve high-resolution multispectral images, pan-sharpening methods aim at fusing the information of low-resolution images. Although most deep learning-based methods can achieve real-time implementation, they do not use all available information inherently contained in the hierarchical nature of the images. The authors of [16] investigated this matter and proposed a real-time multi-scale fusion network that can exploit the information in real time while achieving high performance in the pansharpening process, simultaneously. The proposed network is a type of encoder-decoder neural net and uses coarseto-fine strategy for efficient learning of the features from multi-spectral images.

The authors of [17] investigated the problem of real-time underwater enhancement of super-resolution images. The authors have proposed a two-step method. One step consists of color correction and image quality enhancement. The quality enhancement is applied to compensate hazing, darkening and fogging. The second step consists of resolution optimization enhancement through a convolutional neural net. This improves the robustness of images against different depths and conditions in the water. The experiments demonstrated their effectiveness by quality measures as mean square error, peak signal-to-noise ratio and structural similarity. The authors demonstrate how to achieve real-time performance for their method by reducing the computational complexity of their model.

Paper [18] has investigated the problem of crowd behavior detection in surveillance videos. The conventional approaches consist of a two-stage pipeline that extract lowlevel features and performs classification. Recently, the deep learning techniques have shown high performance compared to conventional approaches. This is essentially a result of to the inherent multi-level feature extraction and representation done by the convolutional neural nets. The authors present a novel integrated handcrafted method based on Conv-LSTM auto-encoder model that is trained by optical flow images. The representation learned at the bottleneck of the architecture is then fed to an encoder and classifier as different branches. Bottleneck is the layer with the lowest number of neurons in every auto-encoder or neural net that creates a low-dimensional representation of the original dataset. This provides the opportunity for learning representation and classification tasks jointly, i.e., the output of the bottleneck is used as input for two different branches where one learns classification and the other learns the reconstruction from the representation.

The authors of [19] investigated the issue of high computational complexity of deep learning models in hyperspectral image analysis. The hyperspectral image is among the wellknown remote sensing imaging that extracts and gathers the data that is not in the visible spectrum. Recently, the deep learning models have improved the efficiency and accuracy of classification in the hyperspectral analysis. However, such models are computationally expensive; hence, making them not eligible for time sensitive tasks. The authors of [19] proposed a convolutional neural network model for hyperspectral imaging tasks that uses principal component analysis as a pre-processing technique to find optimal band extraction for the task.

Paper [20] investigated the issue of degradations of satellite images impacted by the space environment artefacts resulting in blurred images of the earth surface The authors 
propose a neural network method that de-convolves the obtained data to enhance the quality of satellite images. The authors trained the network to approximate the point spread function parameters of satellite optics based on the acquired satellite images during on-orbit operation.

Paper [21] investigated the problem of image de-noising. The proposed approach consists of applying a convolutional neural network $(\mathrm{CNN})$ and then applying anisotropic diffusion (AD). The approach is essentially a hybrid combination of CNN and AD in a multi-level manner. In [22], the authors suggested a CNN-based solution for visual representation in overcrowded scenes. In detail, this research introduces a new architecture, called SD-Net, that provides high-quality maps of density and can count people in real time. The core finding contains minimization of the size of a processing model using the convolutional layers. In fact, the authors have used dilated two-dimensional convolutional layers instead of the conventional pooling layers to reduce the number of the model parameters.

Paper [23] has investigated a joint object detection and segmentation technique for optical remote sensing images taken by cameras placed on drones (unmanned aerial vehicles) to enable the internet of drone concept considering real-time onboard processing at the things layer (read more in [26]). A kind of deep learning method, known as U-Net, has been used as design for the proposed system. The experimental results conducted by the authors show an overall accuracy of at least $92 \%$ for the proposed system in different scenarios.

Paper [24] has worked on a famous machine vision problem for intelligent transportation systems (ITS), i.e., plate recognition. This paper also uses a deep learning-based approach to recognize the Persian and English licences in real-time. The proposed technique achieves an overall accuracy of $99.2 \%$ for plate recognition, and accuracy of $100 \%$ for plate segmentation along with accuracy of $98.41 \%$ for character recognition, and all are performed in real-time. These outputs make it suitable for implementation in Iran ITS industry. The authors of [25] have suggested a deep $\mathrm{Q}$ network for real-time path planning in robotic applications, particularly remote surgery with robots. They used a probabilistic road-map alongside a deep learning network for design of this particular solution. In detail, a robust training approach and a reward-based mechanism have been implemented targeting improved robustness in catastrophic inference while developing the proposed method for dynamic environments.

\section{References}

1. Viola, P., Jones, M.J.: Robust real-time face detection. Int. J. Comput. Vis. 57(2), 137-154 (2004)
2. G.-C. Luh, "Face detection using combination of skin color pixel detection and viola-jones face detector," in 2014 International Conference on Machine Learning and Cybernetics, vol. 1. IEEE, 2014, pp. 364-370.

3. Tavallali, P., Yazdi, M., Khosravi, M.R.: Robust cascaded skin detector based on AdaBoost. Multimedia Tools Appl. 78(2), 2599-2620 (2019)

4. Asadipooya, S. Samadi, M. Moradikia, and R. Mohseni, "Majorization-minimization approach for real-time enhancement of sparsity driven SAR imaging," J. Real-Time Image Process. 2021. [Online]. https://doi.org/10.1007/s11554-021-01076-0

5. P. Gunawardena, O. Amila, H. Sudarshana, R. Nawaratne, A. K. Luhach, D. Alahakoon, A. S. Perera, C. Chitraranjan, N. Chilamkurti, and D. D. Silva, "Real-time automated video highlight generation with dual stream hierarchical growing selforganizing maps," J. Real-Time Image Process., 2021. [Online]. https://doi.org/10.1007/s11554- 020-00957-0

6. Y. He, H. Wang, L. Feng, and S. You, "Motion-blurred star image restoration based on multi-frame superposition under high dynamic and long exposure conditions," J. Real-Time Image Process., 2020. [Online]. https://doi.org/10.1007/ s11554-020-00965-0

7. S. Shivanin, S. C. Patel, V. Arora, B. Sharma, A. Jolfaei, and G. Srivastava, "Real-time cheating immune secret sharing for remote sensing images," J. Real-Time Image Process., 2020. [Online]. https://doi.org/10.1007/s11554-020-01005-7

8. M. K. Moghimi and F. Mohanna, "Real-time underwater image enhancement: a systematic review," J. Real-Time Image Process., 2021. [Online]. https://doi.org/10.1007/ s11554- 020-01052-0

9. Y. Song, J. Qu, and C. Liu, "Real-time registration of remote sensing images with a markov chain model," J. Real-Time Image Process., 2020. [Online]. https://doi.org/10.1007/ s11554- 020-01043-1

10. Y. Song and J. Qu, "Real-time segmentation of remote sensing images with a combination of clustering and Bayesian approaches," J. Real-Time Image Process., 2020. [Online]. https:// doi.org/10.1007/s11554-020-00990-z

11. H. Hassan, A. K. Bashir, M. Ahmad, V. G. Menon, I. U. Afridi, R. Nawaz, B. Luo, "Real-time image dehazing by super-pixels segmentation and guidance filter," J. Real-Time Image Process., 2020. [Online]. https://doi.org/10.1007/s11554-020-00953-4

12. Y. Wu, P. Han, Z. Zheng, "Instant water body variation detection via analysis on remote sensing imagery, J. Real-Time Image Process.," 2021. [Online]. https://doi.org/10.1007/ s11554-020-01062-y

13. M. Das, "Real-time prediction of spatial raster time series: a context-aware autonomous learning model," J. Real-Time Image Process., 2021. [Online]. https://doi.org/10.1007/ s11554-021-01099-7

14. W. Jing, M. Zhang, and D. Tian, "Improved u-net model for remote sensing image classification method based on distributed storage," J. Real-Time Image Process., 2020. [Online]. https://doi. org/10.1007/s11554-020-01028-0

15. H. L. Kennedy, "On the realization and analysis of circular harmonic transforms for feature detection," J. Real-Time Image Process., 2020. [Online]. https://doi.org/10.1007/ s11554-020-01040-4

16. Z. Lai, L. Chen, G. Jeon, Z. Liu, R. Zhong, and X. Yang, "Realtime and effective pan-sharpening for remote sensing using multi-scale fusion network," J. Real-Time Image Process., 2021. [Online]. https://doi.org/10.1007/s11554-021-01080-4

17. M. K. Moghimi and F. Mohanna, "Real-time underwater image resolution enhancement using super-resolution with deep convolutional neural networks," J. Real-Time Image Process., 2020. [Online]. https://doi.org/10.1007/s11554-020-01024-4 
18. F. Rezaei and M. Yazdi, "Real-time crowd behaviour recognition in surveillance videos based on deep learning methods," J. RealTime Image Process., 2021. [Online]. https://doi.org/10.1007/ s11554-021-01116-9

19. A. Mohan and V. M. Sundaram, "V3o2: hybrid deep learning model for hyperspectral image classification using vanilla3d and octave2d convolution," Journal of Real-Time Image Processing, 2020. [Online]. https://doi.org/10.1007/s11554-020-00966-z

20. T. D. Ngo, T. T. Bui, T. M. Pham, H. T. B. Thai, G. L. Nguyen, and T. N. Nguyen, "Image de-convolution for optical small satellite with deep learning and real-time GPU acceleration," J. RealTime Image Process., 2021. [Online]. https://doi.org/10.1007/ s11554-021-01113-y

21. P. Singh and A. Shankar, "A novel optical image denoising technique using convolutional neural network and anisotropic diffusion for real-time surveillance applications," J. RealTime Image Process., 2021. [Online]. https://doi.org/10.1007/ s11554-020-01060-0

22. N. Khan, A. Ullah, I. Haq, V. G. Menon, S. W. Baik, "SD-Net: understanding overcrowded scenes in real-time via an efficient dilated convolutional neural network," J. Real-Time Image Process., 2020. [Online]. https://doi.org/10.1007/ s11554-020-01020-8

23. I. Ahmed, M. Ahmad, G. Jeon, "A real-time efficient object segmentation system based on U-Net using aerial drone images," J. Real-Time Image Process., 2021. [Online]. https://doi.org/10. 1007/s11554-021-01166-Z

24. J. Pirgazi, A. G. Sorkhi, M. M. P. Kallehbasti, "An efficient robust method for accurate and real-time vehicle plate recognition," $J$. Real-Time Image Process., 2021. [Online]. https://doi.org/10. 1007/s11554-021-01118-7

25. P. N. Srinivasu, A. K. Bhoi, R. H. Jhaveri, G. T. Reddy, M. Bilal, "Probabilistic deep Q network for real-time path planning in censorious robotic procedures using force sensors," J. RealTime Image Process., 2021. [Online]. https://doi.org/10.1007/ s11554-021-01122-x

26. Abbasi, M., Yaghoobikia, M., Rafiee, M., Jolfaei, A., Khosravi, M.R.: Efficient resource management and workload allocation in fog-cloud computing paradigm in IoT using learning classifier systems. Comput. Commun. 153, 217-228 (2021)

Publisher's Note Springer Nature remains neutral with regard to jurisdictional claims in published maps and institutional affiliations.

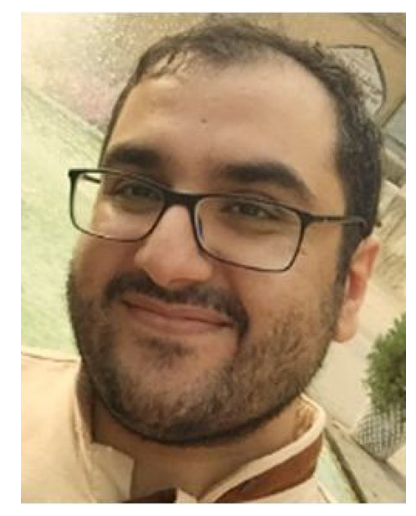

Mohammad R. Khosravi is now with the Department of Computer Engineering, Persian Gulf University, Iran. His main interests include statistical signal and image processing, radar imaging with applications, aerial and satellite remote sensing, computer communications, highperformance computing, internet of things (IoT), cyber-physical systems (CPS), medical bioinformatics, underwater wireless sensor networks, information science and scientometrics. Mohammad has studied electrical engineering with expertise in communications and signal processing for $\mathrm{BSc}, \mathrm{MSc}$ and $\mathrm{PhD}$ degrees.

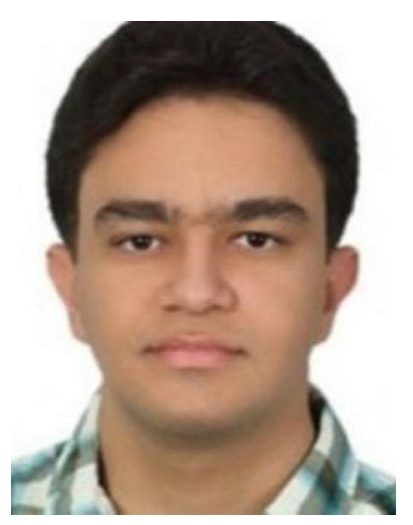

Pooya Tavallali received his B.Sc. and the M.Sc. degrees in Electrical Engineering (Communi-cation Systems) from the Department of Electrical and Electronic Engineering, Shiraz University, Shiraz, Iran, and a PhD from the Department of Electrical Engineering and Computer Science, University of California, Merced, USA. His scientific interest consists of machine learning, statistical signal and image processing, neural networks, statistical pattern recognition and optimization algorithms. 